\title{
Our duties in talent management in the light of the results of the International Hungarian Mathematics Competition of 2017
}

\author{
ERIKa Veres and Katalin Fried
}

Abstract. The 4th International Hungarian Mathematics Competition held in Transcarpathia, Beregszász between April 28 and May 1, 2017, was organized by the Hungarian Carpathian Hungarian Teachers' Association (KMPSZ) and the Ferenc Rákóczi II. Transcarpathian Hungarian Institute (II. RFKMF).

The venue for the competition was the building of the Ferenc Rákóczi II. Transcarpathian Hungarian Institute. 175 students participated in the competition from Hungary, Romania, Serbia, Slovakia and Transcarpathia.

In this article, we are going to deal with the problems given in the two rounds to students in grades 5 and 6 , and, in the light of expectations and performance, we make some suggestions for a more effective preparation of talented students on after-school lessons.

Key words and phrases: Problem solving, achievement control, competition preparation. ZDM Subject Classification: D60, U49.

\section{Introduction}

The International Hungarian Mathematics Competition has been organized for students in grades 9-12 since 1992. In 2014, in Dunaszerdahely (Dunajská Streda) was held a similar competition for students in grades 5-8 and since then every year. The 4th International Hungarian Mathematics Competition held in Transcarpathia, Beregszász between April 28 and May 1, 2017, was organized by the Hungarian Carpathian Hungarian Teachers' Association (KMPSZ) and the 
Ferenc Rákóczi II. Transcarpathian Hungarian Institute (II. RFKMF). The venue for the competition was the building of the Ferenc Rákóczi II. Transcarpathian Hungarian Institute. 175 students participated in the competition, 70 students from Hungary, 40 students from Romania, 20 students from Serbia, 30 students from Slovakia and 15 students from Transcarpathia.

We chose this from the many competitions because we personally participated in all of its phases and we had a chance to examine the works of students.

In this article we are going to deal with the problems given in the two rounds to students in grades 5 and 6 . The analysis of the problems is based on the criteria used by Hoffart (2008), i.e., in the comments we look at the form and content of the problem, examine the problem solving strategies needed to understand and solve the problem, and analyze students' accomplishments. The classification of the representation levels (enactive, iconic and symbolic) is done according Bruner (1966). Finally we make some suggestions for more effective preparation for talented pupils in the light of expectations and performance. The purpose of our suggestions is not giving an idea on how to solve such problems, but to help teachers overcome deficiencies that we found and to strengthen the problemsolving skills.

\section{The tasks}

1. Grade 5, round 1, problem 1 (suggested by Gábor Szücs from Budapest). We are investigating a bank robbery and want to find the robber or robbers. He (or they) used a car to escape with the money stolen. Three suspects were interrogated, namely Andrew, Bart and Chuck. They told that

a) besides Andrew, Bart and Chuck, no other person took part in the robbery,

b) Andrew only works when Chuck works as well,

c) Bert doesn't drive a car.

Whom can we state of for sure that he took part in the robbery?

2. Grade 5, round 1, problem 2 (suggested by Sándor Kántor from Debrecen) Find the least natural number that, written in the decimal system, contains all digits (at least once) and the sum of whose digits is $201 \%$.

3. Grade 5, round 1, problem 3 (suggested by Dr. Miroszláv Sztojka from Beregszász) Four different positive integers, each less than 100, has to be written in place of the letters $A, B, C$ and $D$ in Figure 1 such, that each 
arrow points to a divisor of the number at its starting point. How can you pick these numbers to get $A$ the biggest possible number?

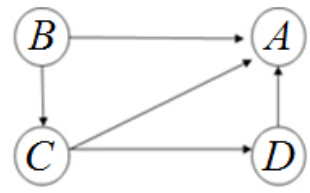

Figure 1

4. Grade 5, round 1, problem 4 (suggested by Erzsébet Csahóczi from Budapest) Lily and Ann are drawing line segments and triangles connecting points on 5 given points.

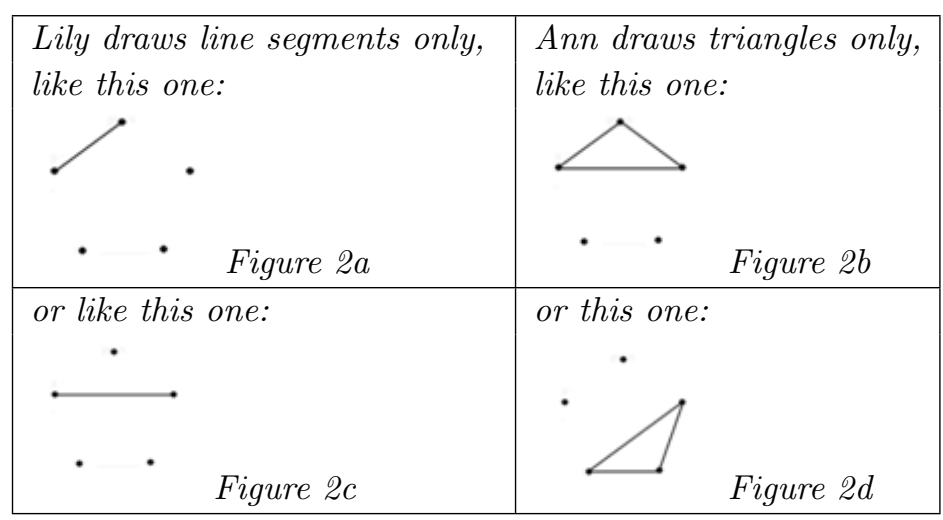

They compete, who can draw more. Drawing all objects, who is going to win?

5. Grade 5, round 2, problem 1 (suggested by István Pécsi from Szolnok) The first International Hungarian Mathematics Competition was organized 25 years ago in Révkomárom (that time for pupils of grade 9-12 only). 1992 is a four digit number whose half and one third is an integer as well. How many four digits numbers are there, whose half and one third are both integers?

6. Grade 5, round 2, problem 2 (suggested by Sándorné Kántor from Debrecen) At the "Namény" Restaurant, the weekly dessert offer is assembled from Sunday morning, picking one from tarts, cakes, ice cream or sweet cream pudding. However, the same dessert should not be served on two consecutive days. The next Friday they have to bake a cake because they have a birthday order. How many different dessert menus can be created for the next week? 
7. Grade 5, round 2, problem 3 (Suggested by Magda Szabó from Szabadka) Show that you can always find two among any 11 positive integers whose difference is divisible by 10 .

8. Grade 5, round 2, problem 4 (Suggested by Mihály Brenyó from Kecskemét) Draw ten different shapes on a square grid whose vertices lie on gridpoints and whose area equals to the area of two squares $\square \square$. Shapes that can be transformed into each other with a move aren't considered different.

9. Grade 6, round 1, problem 1 (Suggested by Sándor Róka from Nyíregyháza) We have written a number on each face of a cube. Numbers on opposite faces add up to the same sum. The three numbers we cannot see are all prime numbers. How big is the largest of them?

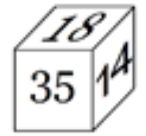

Figure 3

10. Grade 6, round 1, problem 2 (Suggested by Gabriella Ambrus from Budapest) Show that if the sum of two integers end in a zero then the difference of their squares ends in a zero as well.

11. Grade 6, round 1, problem 3. (Suggested by Erzsébet Csahóczi from Budapest) In a family the average age of the children is 11. The oldest child is 17. Without that child, the average age would only be 10. How many children are there in the family? (We consider the ages integers.)

12. Grade 6, round 1, problem 4 (Suggested by Ádám Bessenyei from Budapest) In the isosceles triangle $A B C, A C=B C$ and the vertex angle of it is $30^{\circ}$. Drawing a regular triangle on its leg $B C$ outside, we get triangle $C B D$. Find the angle $D A B$.

13. Grade 6, round 2, problem 1. (Suggested by Magda Szabó from Szabadka) We have 216 pieces of paper. We pick some of them and cut them up into 10 pieces. Then, again we pick some and cut them up into 10 pieces as well. We repeat the same process several times. Then we count the pieces and find that we have 2017 pieces of papers. Can that be right?

14. Grade 6, round 2, problem 2. (Suggested by Erzsébet Csahóczi from Budapest) Each side of the regular triangle DEF was divided into 6 equal parts. Through all division points we drew lines parallel to the sides of the triangle. The area of smallest triangle determined by the intersection points of these lines is $1 \mathrm{~cm}^{2}$.

a) Find the area of triangle $D E F$ in $\mathrm{cm}^{2}$ 's. 
b) Let the dividing point closest to vertex $D$ on the side $D E$ be denoted by $A$, the trisecting point farthest from vertex $D$ on side $D F$ by $B$ and the bisecting point of side $E F$ by $C$. Find the area of triangle $A B C$ in $\mathrm{cm}^{2}$ 's.

15. Grade 6, round 2, problem 3. (Suggested by László Egyed from Baja) Peti forgot the 7-digit phone number of his friend, remembering only the following. All digits are different, there is no 0 among the digits, but there is a digit 1. The first three digits form a number divisible by 9 , and the numbers come in increasing order such, that the differences of two consecutive numbers are the same. The phone number itself is divisible by 5. According to these conditions, at most how many calls does Peti have to make to reach his friend, trying each possibility at most once?

16. Grade 6, round 2, problem 4. (Suggested by Béla Kovács from Szatmárnémeti) Find all two-digit natural numbers whose square ends in the same twodigit number as the number itself. Are there any three-digit natural numbers whose square ends in the same three-digit number as the number itself?

\section{Analysis of the tasks}

The graph in Figure 4 shows the success of all participants and those from the Transcarpathians, according to problems and the item difficulty index (i.e. D difficult under $25 \%$, M moderately difficult between $25 \%$ and $75 \%$, E easy over $75 \%)$.

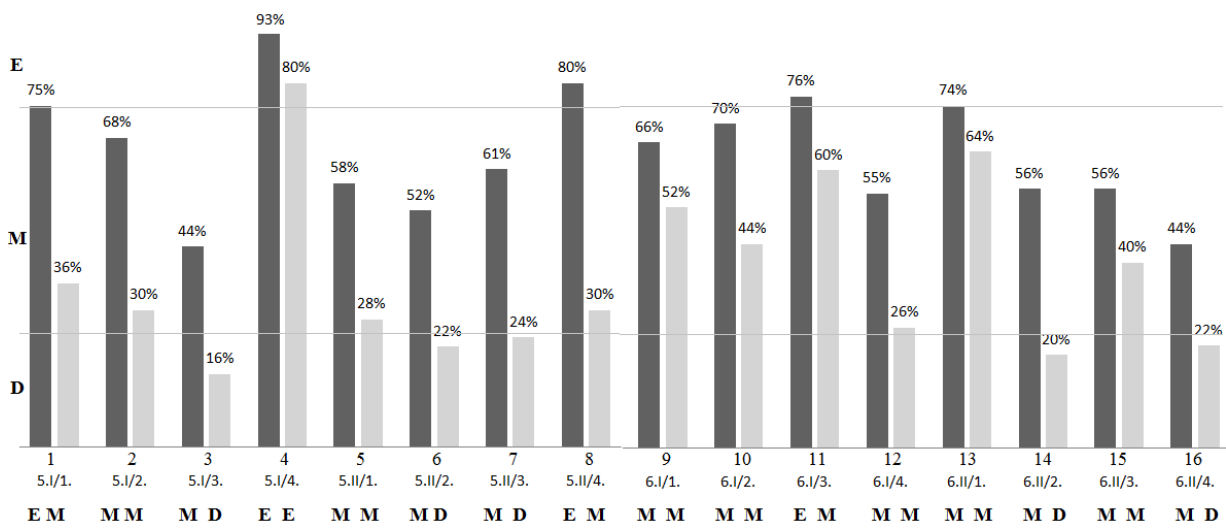

Figure 4. The performance of fifth and six-graders; all participants (black) and Transcarpathian participants (gray) per problem. 
The performance of Hungarian students in Transcarpathia is below the average in all tasks. In order to find (at least in part) the reasons for this we

- analyzed the tasks (the formulation of the problem, the necessary knowledge elements to understand the problem, the expected solution, the competencies needed for the solution);

- compared the Ukrainian and Hungarian curricula and textbooks;

- looked at the students' work.

Now we show the analysis of 1-1 task from both age groups and both rounds, in which the performance of the Transcarpathians was far behind the performance of the whole field.

\section{Remarks to task 3}

A. The problem is presented at verbal, visual and symbolic representational level at the same time. The figure consists of a directed graph to help avoid explaining overcomplicated relations; its task is to present the problem in a more compact way. The vocabulary and word usage correspond to the age. The expression "positive numbers less than 100" was written in a dialect ("less from 100"), but this did not seem to bother the Hungarian students and was familiar to the Transcarpathians.

B. The necessary knowledge elements to understand the problem are " $a$ being a divisor of $b$ " and " $b$ being the multiple of $a$ " and knowing the transitivity of the relations "being a divisor of" and "being a multiple of" and being able to interpret the corresponding relations from the graph.

C. The basic idea of the solution expected is the from the graph we can read a divisibility-chain: $A|D| C \mid B$, from which we can see that $A$ is the biggest possible number if $D: A, C: D, B: C$ are as little as possible, that is, 2 . Then from $B=2 \cdot C=4 \cdot D=8 \cdot A$ we get that since $B<100, A$ is at most 12. In that case, $D=24, C=48$ and $B=96$.

D. To find the idea of the solution, we have to discover that conditions $B \rightarrow A$ and $C \rightarrow A$ follow from the relation-chain $B \rightarrow C \rightarrow D \rightarrow A$. Introducing new notations to avoid division, we can simplify the written form of the solution though the divisor-multiple relation is usually written in the form of factors of a number. Rewriting the relation chain of divisibility to equality can be an interesting discovery:

$$
B=i \cdot C=i \cdot(j \cdot D)=i \cdot j \cdot(k \cdot A)=i \cdot j \cdot k \cdot A,
$$


from which we can see that $A$ will be the biggest possible if the multipliers $i, j, k$ are the least possible, that is, 2 . Finding the biggest number $A$ satisfying the condition $8 A<100$ can be done several ways.

E. The solution rate of the problem is $44 \%$, so it can be considered a moderately difficult one. It was definitely difficult for Transcarpathian pupils, where the solvency was $16 \%$.

F. In view of the tasks, it is conspicuous that the parallel use of the different representations and the movement between the representational levels also confused the participants from the Transcarpathians, because they had less emphasis on linking textual, graphic and symbolic representations (Figure 5a). On Figure 5b we show a part of the work of a pupil who interpreted correctly the text and the conditions and could make a difference between divisor and multiple and could express it on his or her drawing. He/she solved the problem by the trial and error method. On Figure $5 \mathrm{c}$ one can see essentially the expected solution.
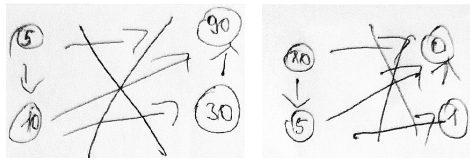

Figure 5 a

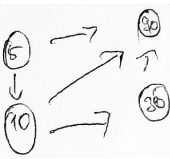

(36)

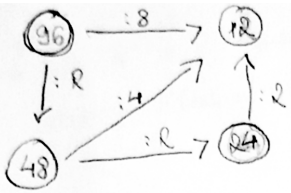

Figure $5 b$

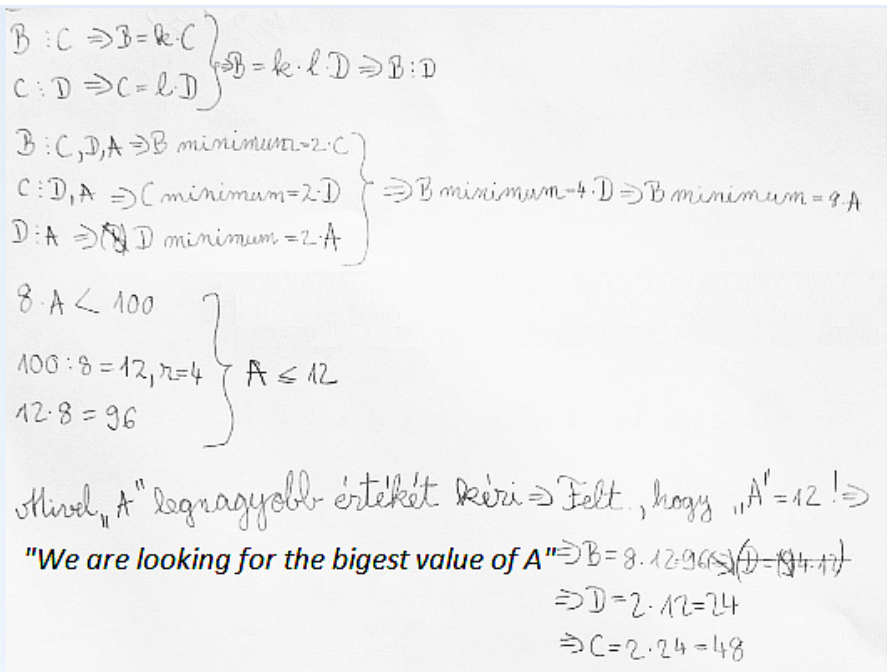

Figure 5c 


\section{Remarks to task 8}

A. The problem is put the way it is usual in textbooks, with the combination of textual and symbolic representations. It is a construction type of open problem in the sense that it has infinitely many solutions, from which 10 examples have to be given by drawing.

B. The necessary knowledge elements to understand the problem are coordination skills on the grid, and the concept of grid-point as a mathematical term and area.

C. The basic idea of the expected solution is that one can combine and draw further polygons from others, whose area are known or can be calculated easily.

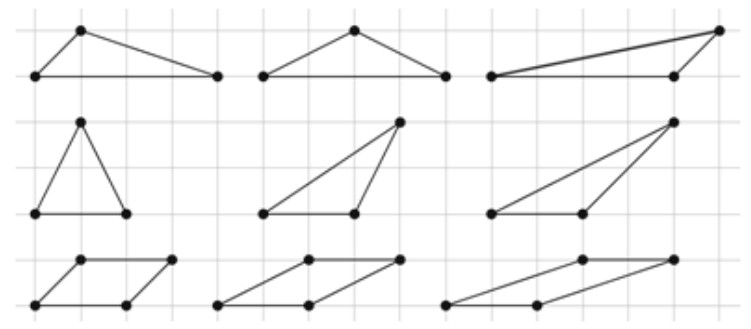

Figure 6

D. The necessary skills to find the idea of the solution are based on the area relative to the area of the unit, and dissection. Those who can find two solutions might easily find ten. It is difficult to prove though, that these solutions are correct. Dissection cannot always be seen. It is important to disregards the fact that the area is given by two connecting squares.

E. The problem proved to be easy, the solvency was $80 \%$, however, it was only $30 \%$ amongst the pupils from Trancarpathians.

F. There was a student, who described the shapes with text. Another drew 45 shapes, among which 16 were correct. In Figure 7 one can see the how the Transcarpathian students tried to find the shapes. 

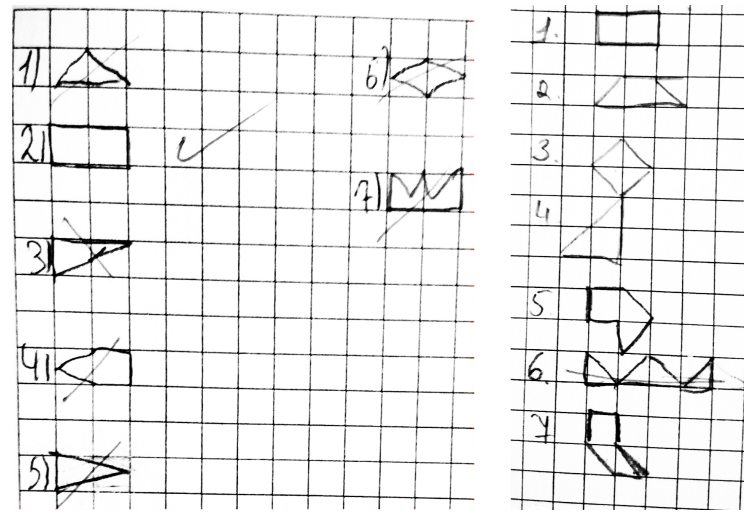

Figure 7

Remarks to task 12

A. The problem is given with the combination of textual and symbolic representations, but without a figure. In precise mathematical language, it is difficult to interpret the expression "outside". This, however, didn't seem to be disturbing, as the majority of the students started the solution by drawing a picture, anyway (Figure 8).

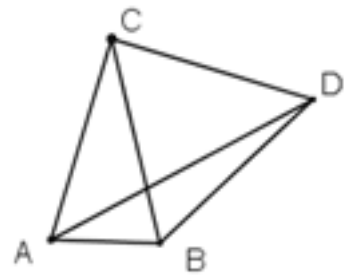

Figure 8

B. The required knowledge elements to understand the problem are the usual notations of the triangle, understanding concepts isosceles and regular, drawing a triangle on a leg (outside) and the notation of "angle $D A B$ ".

C. The basic idea of the expected solution is calculating the angles. Since the vertex angle equals $30^{\circ}$, the base angles equal $75^{\circ}$. The angles of the regular triangle equal $60^{\circ}$ and all its sides are of equal length, therefore triangle $A C D$ is an isosceles triangle $(A C=C D)$, its angle at vertex $C$ is $60^{\circ}+30^{\circ}=90^{\circ}$. The triangle $A C D$ is an isosceles right triangle. Its angles at both vertices $A$ and $D$ equal $45^{\circ}$. So angle $D A B$ is equal to $75^{\circ}-45^{\circ}=30^{\circ}$. 
D. The solution requires frequent point shifting from the angles of the special triangles to its sides and from the sides to the angles.

E. The task is moderately difficult, its solvency is $55 \%$, but is only $26 \%$ for Transcarpathians pupils.

F. Some Transcarpatian students have been stuck on the interpretation of the text, which is the vertex angle (Figure 9a), or the notation of "angle $D A B$ " (Figure 9b).

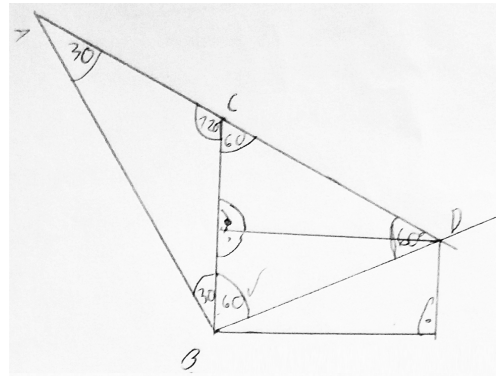

Figure $9 a$

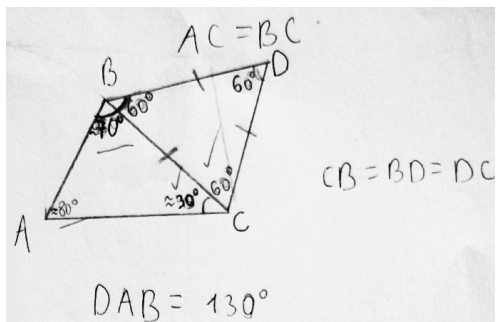

Figure $9 b$

Remarks to task 14

A. The problem is put the way it is usual in textbooks, with the combination of textual and symbolic representations. The two questions (apart from referring to the same triangle with subdivided sides) are independent from each other.

B. To understand the problem one requires to understand some basic geometric concepts, and the measures of area.

C. The basic thought of the expected solution of question is finding a strategy to actually count the area on a figure: $1+3+5+7+9+11=36 \mathrm{~cm}^{2}$. The area of triangle $A B C$ can be calculated by dissecting the triangle (as shown on the Figure 10) and complement the sub triangles into parallelograms: $2+3+6=11 \mathrm{~cm}^{2}$. 


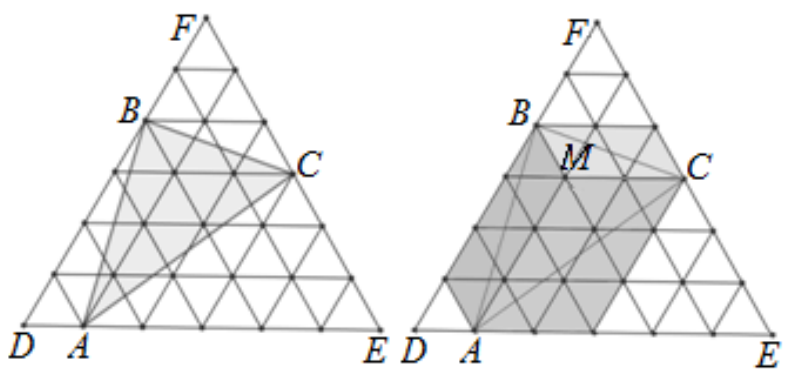

Figure 10

D. To find the idea of the solution, a proficiency of area measurement comparison, dissections and completion, the ability to move from one representation to another, to visualize according to text and the perception of part and whole is necessary.

E. The problem is moderately difficult, its solvency is $56 \%$, while only $20 \%$ among pupils from the Transcarpathians.

F. The majority was able to solve the task a), but some students had problem with the interpretation of the text and stalled while drawing the picture (Figure 11a). There were also students who solved the problem using dissection (Figure 11b).

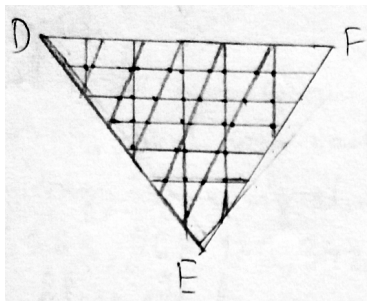

Figure $11 a$

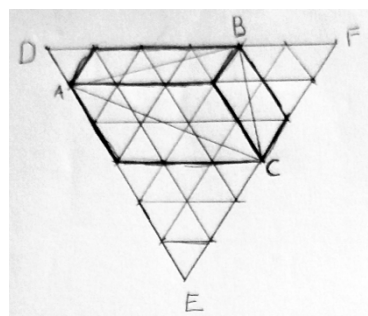

Figure $11 b$

Suggestions for Hungarian after-school enrichment programs for talented pupils in the Transcarpathians

We are trying to find an explanation (at least in part) for the weaker performance of the Transcarpathian participants, and we suggest some processing methods and tasks to eliminate the problems. We focus on raising the problem-solving 
skills to a higher level rather than on solving the problems of the competition in question.

\section{Developing the network of knowledge}

Some of the differences in performance can be explained by differences in content and order of materials in the curriculum. This is a feature that is difficult to change, but we draw the attention to some possibilities.

Tamás Varga's complex program of mathematics education had building concepts gradually, from concept stem to precise mathematical concept as a central element of it, as it has an important role in engraving and integrating (Varga 1966). At least on after-school enrichment programs we should pay more attention to the principle of spiralism. It is necessary to strive for knowledge and procedures to be incorporated horizontally and vertically into the knowledge network.

The result of Example 12 can be partly explained by the fact that the concepts of special types of triangles are based on the classification of these by sides (isosceles, equilateral or regular) and by largest angles (acute, right, obtuse) are handled separately in the textbook, independently of the connections between sides and angles. It is a good idea to bring these two approaches closer on afterschool lessons.

The extreme difficulty of Example 3 can be explained by the following: In Hungary, the concepts even, odd, composite, prime, divisor, multiple are discussed from grade 1 to grade 6 . In mathematics education in Ukraine, pupils in class 6 are confronted consecrated with the topic of divisibility. In our opinion, the notion of $A \mid B$ was not sufficiently practiced for pupils of Transcarpathians, the expression "each arrow points to a divisor of the number at its starting point" must have been a novelty, moreover, the text referred to a divisor, while in the graph the direction of the arrow referred to multiple, so the notation $B \rightarrow A$ meant $B$ is a multiple of $A$. Divisor pairs play an important role in divisibility and in finding the prime factorization of a number. Thinking about divisor pairs helps not only finding prime factorization faster, but it also helps to separate the concept of divisor in divisibility from divisor in the operation of dividing. It is a good idea to draw the attention of pupils to the divisor pairs and the prime factor form of numbers on after-school lessons. A great number of such types of exercises can be found in Hungarian textbooks, workbooks and problem books.

In view of the tasks, it is conspicuous that the parallel use of the different representations and the movement between the representational levels also confused 
the participants from the Transcarpathians, because they had less emphasis on linking textual, graphic and symbolic representations.

\section{Developing the comprehension and interpretation of texts}

Another possible component of weaker performance is that the development of reading comprehension and interpretation of texts is not included among the central objectives of mathematics learning; however the Hungarian students living in the minority would need it. You can develop text comprehension and text interpretation with introductory, tune-in, tasks. You can recall or create such phrases, concepts, notations, relationships, procedures that can help the pupils in solving the given problem. In the process of problem solving, questions helping clarification serve as the purpose of checking understanding and directing attention. (Ambrus, Vásárhelyi 2016). It is worth to convert text from every day speech to the concise form of competitions gradually. This principle should apply in the wording of introductory, tune-in tasks as well. It is a good idea to give time practicing text comprehension and interpretation in these problems as well. It is advisable to prepare the tasks also in a version that supports text comprehension and interpretation (version a) and b) in the examples). If the students do not feel the difference between the two approaches in the given topic, then the comprehension task can be omitted and we can directly focus on the problem solving.

\section{Developing the problem solving ability}

Independently of the curriculum, it is worth to practice

- thinking methods (relations, quantifiers, conclusion chain, indirect thinking),

- problem solving strategies (systematic trial and error method, thinking backwards, pigeonhole principle),

- basic concepts of elementary number theory (different forms of numbers, divisor, multiple, divisor pairs, divisibility of sums/differences, products, connection between divisibility and number systems),

- basic concepts of elementary geometry (interpreting figures, drawing figures according to the text, geometric constructions, dissections),

- data processing, basics of statistics (ordering data, average, smallest, greatest). 
The team of the a group of teachers teaching mathematics in Hungarian language in Transcarpathia collects problem series in each topic mentioned above for the after-school lessons. (Regarding the method of thinking backwards see for example Veres 2016). In this work we used some ideas from the Hungarian textbooks, problem books and methodological literature. We refer to a few Hungarian sources available electronically: for example Fried at al 2013, Hraskó at al 2013, Wintsche 2016.

\section{Examples}

Due to space limitation we show here only a few examples as illustration. Concepts of divisibility, divisor, multiple, divisor pairs

1. Collect the divisors of numbers $8,10,18$ and 19. Which number had the most (less) divisors? Find a positive integer that has exactly five positive divisors. (Wintsche 2016, page 37)

2a Find the largest positive integer less than 100 which, when divided by 6 gives a remainder divisible by 5 .

$2 \mathrm{~b}$ List all positive integers less than 100 divisible by both 5 and 6 . Which is the largest of these?

2c Write divisibility statements about these numbers using the textual form " $A$ is a divisor of $B$ ", " $B$ is a multiple of $A$ ", the relational notation $A \mid B$, the form of equality like $B=k \cdot A$. Replace $\mathrm{A}$ and $\mathrm{B}$ by appropriate numbers and read the relationships $B \rightarrow A$ and $A \leftarrow B$ in several ways, if the arrow connects a divisor and a multiple by pointing to the divisor of the number at its starting point!

3a Find the larges positive integer less than 50 that has exactly two positive divisors.

3b List all the positive integers less than 50 that has exactly two positive divisors. Which is the largest of them?

3c Write the divisibility relation between these numbers by using the textual forms, the relational notation and the equality form.

4a Write three different positive integers less than 100 in the circles in Figure 12 in place of the letters $A, B$ and $C$ such, that each arrow should point to the divisor to the number at its starting point. How should you choose these numbers to make the one at letter $A$ be the largest possible? 


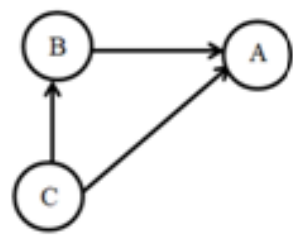

Figure 12

4b Write different positive integers less than 100 in the circles in Figure 12 in place of the letters such, that each arrow should point to the divisor to the number at its starting point. How do the number have to be chosen to make the one at letter $A$ be the largest possible? Is there an arrow which doesn?t play any role in your solution?

Remark: Although in the text the word "divisor" is written, but the interpretation of the relation " $C \rightarrow B$ " is more useful in the form " $C$ is multiple to $B$ ". It is also important to use the usual mathematical symbols besides the occasional notation.

\section{Thinking backwards}

5. So many wanted to enter a cross-country race that the organizers decided to split up the participants in groups. First, they let one part of the racers start, then every ten minutes they would let start one quarter of the contestants of the previous group. There were 5 racers in the last group. How many participants were there all together, if we know that the number of the participants has 4 digits and is divisible by 3 ?

Remark: If we consider the rounds backward, then the number of competitors will be quadrupled in each round. The number of contenders:

\begin{tabular}{|c|c|c|c|c|c|c|}
\hline In the actual round & 5 & 20 & 80 & 320 & 1280 & 5120 \\
\hline All together & 5 & 25 & 105 & 425 & 1705 & 6825 \\
\hline
\end{tabular}

There is no need to go on with the sequence, because the next terms will have 5 digits. 1705 has 4 digits, but it is not divisible by 3 . 6825 has 4 digits and is divisible by 3 . The number of participants is 6825 .

\section{Area measurement comparison, dissections and completion}

For the first steps we can use peg board, tangram or pentomino. We can create interesting shapes with a rubber ring or thread. The area can be determined by completing or splitting. We can compare the area of triangles of same basis and equal height, and so on (Figure 13a). 
6. Find the area of the square $A B C D$ in Figure 13b. We pick grid the points $E, F, G, H$ on the perimeter of the square $A B C D$. What proportion of the are of the square $A B C D$ is the area of the quadrilateral $E F G H$ ?

Create your own problem! For example, finish the question: "How to pick the grid points $E, F, G, H$ on the perimeter of the square $A B C D$ to make the area of the quadrilateral ?"

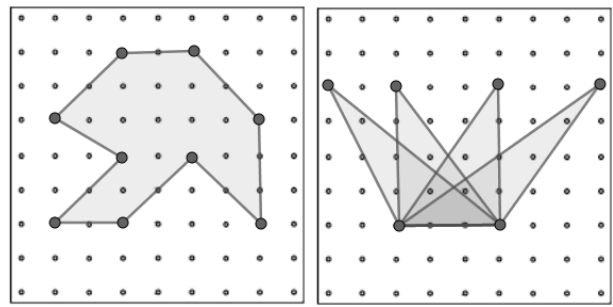

Figure $13 a$

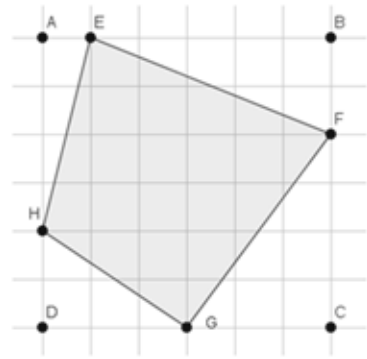

Figure $13 b$

\section{References}

[1] A. Ambrus, É. Vásárhelyi, Dividing a square: Is it possible? Tray to do it!, in: Problem Solving in the Mathematics Classroom: Perspectives and Practices from Different Countries, (T. Hodnik-Čadež, A. Kuzle, B. Rott, eds.), WTM Verlag, Münster, 2016, 61-72.

[2] J. Bruner, The Process of Education, Revised Edition Harvard University Press, 1977.

[3] K. Fried, J. Korándi, J. Török, Elemi matematika példatár tanároknak, Elektronikus jegyzet. TÁMOP-4.1.2.B.2-13/1-2013-0007 Országos koordinációval a pedagógusképzés megújításáért, 2013

http://ttomc.elte.hu/sites/default/files/kiadvany/tamop_elemi.pdf.

[4] E. Hoffart, Analysen zu den Aufgaben der Orientierungsarbeit in Hessen 2005, Beiträge zum Mathematikunterricht (2008), 286-289 http://www.mathematik. uni-dortmund.de/ieem/BzMU/ BzMU2008/BzMU2008/BzMU2008_HOFFART_Eva_CD.pdf.

[5] Hegyvári, J. Korándi, J. Török, Elemi matematika feladatgyűjtemény, (A. Hraskó, ed.), 2013

http://www.tankonyvtar.hu/hu/tartalom/tamop412A/

2011-0064_71_matematika_elemi_matematika_feladatgyujtemeny/adatok.html. 
[6] T. Varga, Complex method of teaching mathematics to children from the age of 6 years. Contemporary methods and tools in the service of school reform, Tankönyvkiadó Budapest, Hungary, 1966.

[7] E. Veres, Backwards is sometimes better, 2016 https://prezi.com/hy7kpuejared/visszafele-neha-jobb/.

[8] E. Veres, K. Fried, Suggestions for talented pupils in the Transcarpathians, 2017 http://mathdid.elte.hu/html/forumtartalom.html\#vtn2017.

[9] G. Wintsche (ed.), Matematika 5-8. [Mathematics 5-8. Textbooks], Oktatáskutató és Fejlesztó Intézet. Retrieved from https://player.nkp.hu.

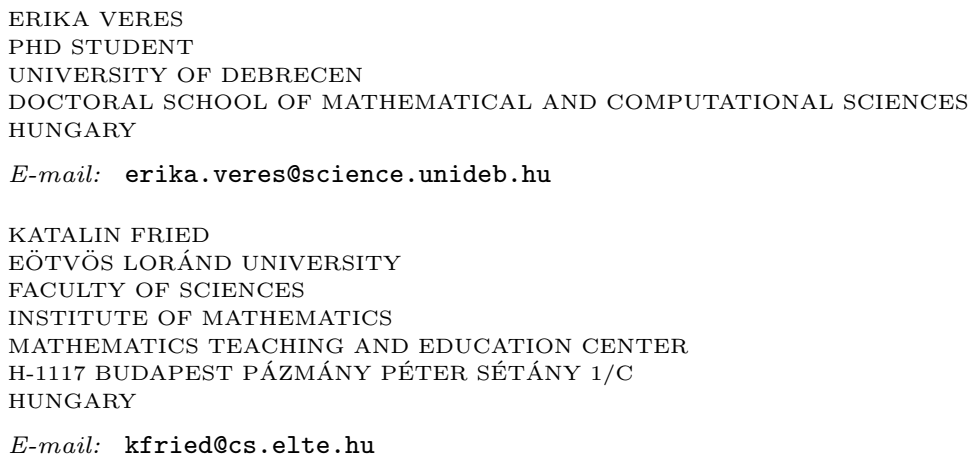

(Received December, 2017) 